Peter E. Kloeden Eckhard Platen

\title{
Numerical Solution of Stochastic Differential Equations
}

With 85 Figures

Springer 


\section{Contents}

Suggestions for the Reader ................. xvii Basic Notation . . . . . . . . . . . . . . . . . xxi Brief Survey of Stochastic Numerical Methods ........... . xxiii

\section{Part I. Preliminaries}

Chapter 1. Probability and Statistics .............. 1

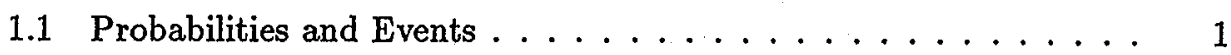

1.2 Random Variables and Distributions . . . . . . . . . . . 5

1.3 Random Number Generators ... . . . . . . . . . . . 11

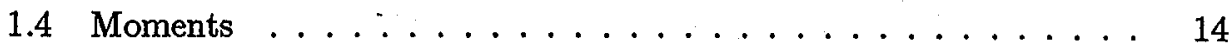

1.5 Convergence of Random Sequences .............. 22

1.6 Basic Ideas About Stochastic Processes . . . . . . . . . . . 26

1.7 Diffusion Processes ..................... 34

1.8 Wiener Processes and White Noise . . . . . . . . . . . 40

1.9 Statistical Tests and Estimation .............. 44

Chapter 2. Probability and Stochastic Processes . . . . . . . 51

2.1 Aspects of Measure and Probability Theory . . . . . . . . . 51

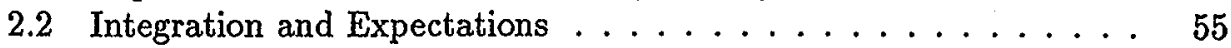

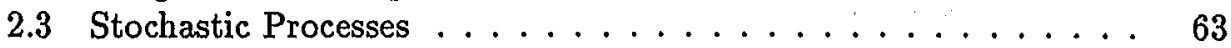

2.4 Diffusion and Wiener Processes ................. 68

\section{Part II. Stochastic Differential Equations}

Chapter 3. Ito Stochastic Calculus . . . . . . . . . . 75

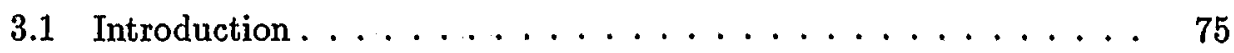

3.2 The Ito Stochastic Integral . . . . . . . . . . . . . 81

3.3 The Ito Formula . . . . . . . . . . . . . . . . . . . 90

3.4 Vector Valued Ito Integrals . . . . . . . . . . . . . . . 96

3.5 Other Stochastic Integrals . . . . . . . . . . . . . 99

Chapter 4. Stochastic Differential Equations ......... 103

4.1 Introduction . . . . . . . . . . . . . . 103

4.2 Linear Stochastic Differential Equations . . . . . . . . . . . 110 
4.3 Reducible Stochastic Differential Equations . . . . . . . . . . 113

4.4 Some Explicitly Solvable Equations . . . . . . . . . . . . . . 117

4.5 The Existence and Uniqueness of Strong Solutions . . . . . . . . 127

4.6 Strong Solutions as Diffusion Processes . . . . . . . . . . . 141

4.7 Diffusion Processes as Weak Solutions . . . . . . . . . . . . . . 144

4.8 Vector Stochastic Differential Equations . . . . . . . . . . . 148

4.9 Stratonovich Stochastic Differential Equations . . . . . . . . . . 154

Chapter 5. Stochastic Taylor Expansions _ . . . . . . . 161

5.1 Introduction . . . . . . . . . . . . . . . . 161

5.2 Multiple Stochastic Integrals $\ldots \ldots \ldots \ldots \ldots \ldots \ldots$

5.3 Coefficient Functions . . . . . . . . . . . . . . . 177

5.4 Hierarchical and Remainder Sets . . . . . . . . . . . . 180

5.5 Ito-Taylor Expansions . . . . . . . . . . . . . . . . . . . . 181

5.6 Stratonovich-Taylor Expansions . . . . . . . . . . . . . . 187

5.7 Moments of Multiple Ito Integrals _. . . . . . . . . . . 190

5.8 Strong Approximation of Multiple Stochastic Integrals . . . . . . 198

5.9 Strong Convergence of Truncated Ito-Taylor Expansions . . . . . . 206

5.10 Strong Convergence

of Truncated Stratonovich-Taylor Expansions . . . . . . . . 210

5.11 Weak Convergence of Truncated Ito-Taylor Expansions . . . . . 211

5.12 Weak Approximations of Multiple Ito Integrals . . . . . . . . . 221

\section{Part III. Applications of Stochastic Differential Equations}

Chapter 6. Modelling with Stochastic

Differential Equations $\ldots \ldots \ldots \ldots \ldots$. . . . . . . . 227

6.1 Ito Versus Stratonovich . . . . . . . . . . . . . . . . . . 227

6.2 Diffusion Limits of Markov Chains . . . . . . . . . . . . 229

6.3 Stochastic Stability . . . . . . . . . . . . . . . . . 232

6.4 Parametric Estimation . . . . . . . . . . . . . . . . 241

6.5 Optimal Stochastic Control . . . . . . . . . . . . . . . . . . 244

6.6 Filtering . . . . . . . . . . . . . . . . . 248

Chapter 7. Applications of Stochastic Differential Equations . 253

7.1 Population Dynamics, Protein Kinetics and Genetics . . . . . . . 253

7.2 Experimental Psychology and Neuronal Activity . . . . . . . 256

7.3 Investment Finance and Option Pricing . . . . . . . . . 257

7.4 Turbulent Diffusion and Radio-Astronomy . . . . . . . . . . . 259

7.5 Helicopter Rotor and Satellite Orbit Stability . . . . . . . 261

7.6 Biological Waste Treatment, Hydrology and Air Quality . . . . . 263

7.7 Seismology and Structural Mechanics _. . . . . . . . . 266

7.8 Fatigue Cracking, Optical Bistability and Nemantic Liquid Crystals . . . . . . . . . . . . . . . . . 269

7.9 Blood Clotting Dynamics and Cellular Energetics . . . . . . . . 271 
7.10 Josephson Tunneling Junctions, Communications and Stochastic Annealing . . . . . . . . . . . . 273

\section{Part IV. Time Discrete Approximations}

Chapter 8. Time Discrete Approximation of Deterministic Differential Equations

8.1 Introduction . . . . . . . . . . . . . . . . . . . 277

8.2 Taylor Approximations and Higher Order Methods . . . . . 286

8.3 Consistency, Convergence and Stability . . . . . . . . . 292

8.4 Roundoff Error . . . . . . . . . . . . . . . . . . . 301

Chapter 9. Introduction to Stochastic

Time Discrete Approximation . . . . . . . . . . . . 305

9.1 The Euler Approximation . . . . . . . . . . . . . . . 305

9.2 Example of a Time Discrete Simulation . . . . . . . . . . 307

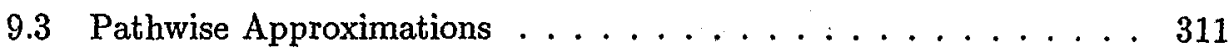

9.4 Approximation of Moments . . . . . . . . . . . 316

9.5 General Time Discretizations and Approximations . . . . . . . . 321

9.6 Strong Convergence and Consistency . . . . . . . . . . . 323

9.7 Weak Convergence and Consistency ... . . . . . . 326

9.8 Numerical Stability . . . . . . . . . . . . . . . 331

Part V. Strong Approximations

Chapter 10. Strong Taylor Approximations _. . . . . . . 339

10.1 Introduction . . . . . . . . . . . . . . . . 339

10.2 The Euler Scheme . . . . . . . . . . . . . . . 340

10.3 The Milstein Scheme . . . . . . . . . . . . . . 345

10.4 The Order 1.5 Strong Taylor Scheme . . . . . . . . . . 351

10.5 The Order 2.0 Strong Taylor Scheme . . . . . . . . . . 356

10.6 General Strong Ito-Taylor Approximations . . . . . . . . . 360

10.7 General Strong Stratonovich-Taylor Approximations . . . . . . 365

10.8 A Lemma on Multiple Ito Integrals . . . . . . . . . . 369

Chapter 11. Explicit Strong Approximations $\ldots \ldots \ldots \ldots 373$

11.1 Explicit Order 1.0 Strong Schemes $\ldots \ldots \ldots \ldots \ldots$

11.2 Explicit Order 1.5 Strong Schemes . . . . . . . . . . . 378

11.3 Explicit Order 2.0 Strong Schemes . . . . . . . . . 383

11.4 Multistep Schemes . . . . . . . . . . . . . . 385

11.5 General Strong Schemes . . . . . . . . . . . . . 390 
Chapter 12. Implicit Strong Approximations . . . . . . . . 395

12.1 Introduction . . . . . . . . . . . . . . . . . . 395

12.2 Implicit Strong Taylor Approximations . . . . . . . . . . . . . 396

12.3 Implicit Strong Runge-Kutta Approximations . . . . . . . . . . . . 406

12.4 Implicit Two-Step Strong Approximations . . . . . . . . . . . 411

12.5 A-Stability of Strong One-Step Schemes . . . . . . . . . . 417

12.6 Convergence Proofs . . . . . . . . . . . . . 420

Chapter 13. Selected Applications

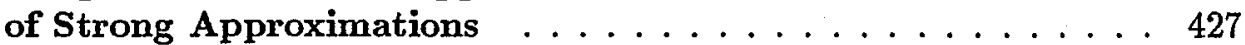

13.1 Direct Simulation of Trajectories . . . . . . . . . . . . . . 427

13.2 Testing Parametric Estimators . . . . . . . . . . . . . 435

13.3 Discrete Approximations for Markov Chain Filters . . . . . . . 442

13.4 Asymptotically Efficient Schemes . . . . . . . . . . . . . 453

\section{Part VI. Weak Approximations}

Chapter 14. Weak Taylor Approximations . . . . . . . . . 457

14.1 The Euler Scheme . . . . . . . . . . . . . . . 457

14.2 The Order 2.0 Weak Taylor Scheme . . . . . . . . . . . . 464

14.3 The Order 3.0 Weak Taylor Scheme . . . . . . . . . . . . 468

14.4 The Order 4.0 Weak Taylor Scheme . . . . . . . . . . . 470

14.5 General Weak Taylor Approximations . . . . . . . . . . . . 472

14.6 Leading Error Coefficients . . . . . . . . . . . . . . . . . . . 480

Chapter 15. Explicit and Implicit Weak Approximations _. . 485

15.1 Explicit Order 2.0 Weak Schemes . . . . . . . . . . . . . 485

15.2 Explicit Order 3.0 Weak Schemes . . . . . . . . . . . . . . 488

15.3 Extrapolation Methods . . . . . . . . . . . . . . . . 491

15.4 Implicit Weak Approximations . . . . . . . . . . . . . . 495

15.5 Predictor-Corrector Methods . . . . . . . . . . . . . . 501

15.6 Convergence of Weak Schemes $\ldots \ldots \ldots \ldots$. . . . . 506

Chapter 16. Variance Reduction Methods . . . . . . . . . . 511

16.1 Introduction . . . . . . . . . . . . . . . . . . . 511

16.2 The Measure Transformation Method . . . . . . . . . . . . 513

16.3 Variance Reduced Estimators . . . . . . . . . . . . . . . . 516

16.4 Unbiased Estimators . . . . . . . . . . . . . . . . . . . . 522

Chapter 17. Selected Applications of Weak Approximations . 529

17.1 Evaluation of Functional Integrals . . . . . . . . . . . . . 529

17.2 Approximation of Invariant Measures . . . . . . . . . . . 540

17.3 Approximation of Lyapunov Exponents . . . . . . . . . . . 545 
Solutions of Exercises $\ldots \ldots \ldots \ldots \ldots \ldots$. . . . . . . . . 549 Bibliographical Notes . . . . . . . . . . . . 587 Bibliography . . . . . . . . . . . . . . . . . . 599 Index ......................... 629 